\title{
Multipurpose Low-Cost Humanoid Platform and Modular Control Software Development
}

\author{
Filipe Silva and Vítor Santos \\ University of Aveiro \\ Portugal
}

\section{Introduction}

Humanoid robotics is becoming quite popular especially after Honda's robots in the 90s' and their evolution ever since (Hirai et al., 1998; Sakami et al., 2002). In the meantime, other research groups have developed their own humanoid platforms making them available commercially for both the edutainment or academic communities (Furuta et al., 2001; Lohmeier et al., 2004; Kaneko et al., 2004; Kim et al., 2004; Nagasaka et al., 2004). Valuable and versatile platforms for research, however, still imply prohibitive costs for most small research groups wanting to perform activity on humanoid balance, walk and advanced perception, plus the difficulty associated to full autonomy, which is the ultimate requisite. Several initiatives to develop commonly accessible and open platforms for research and development have appeared, such as the well-known OpenPino project (Yamasaki et al., 2000). Nonetheless, for several reasons, possibly technological issues, these open platforms did not surge as massively as would be expected. Another possible reason is that some - academic groups prefer rather to develop a platform themselves since that creates E opportunities to teach engineering for students. The combination of these reasons led the O. authors to gradually start a project, in early 2004, to develop a humanoid platform with $\stackrel{\Xi}{\subseteq}$ off-the shelf low-cost components, but baring in mind the needs for versatility and full 을 autonomy so as to comply with the RoboCup regulations and, eventually, enter the 들 competition when possible. The topics covered in this chapter are largely inspired by the research work behind this project, namely the major design concerns and the development of the modular low-level control architecture. Special emphasis is given to the distributed local control to achieve proper adaptation to real-world uncertainties in dynamic $\$$ environments. The text seeks a balance between the application of various technical O solutions to a practical engineering system, and a mathematically rigorous theoretical

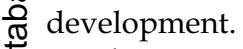

त्ञ In what concerns the humanoid system, the actuators, sensors, and mechanical components is were selected and configured to devise the platform main structure (the hardware includes \& 22 DOFs); that accounts for mechanical axles and links, servomotor typology, gear units, $\underset{\&}{0}$ adequate Ion-Li battery packs, among others. For enhanced control power and versatility, the entire system is based on a distributed architecture supported on a Controller Area ฮั Network (CAN) arrangement of PIC microcontrollers with local decision capabilities, but O able to comply with global directives issued by a central unit based on an embedded PC 
system. Still sticking to low cost, proper electronics and signal conditioning were also developed; that includes force sensors for the feet that were custom-developed and calibrated for the force-based control developed latter. RC servomotors are a low-cost full integrated solution, but they offer too simple control schemes allowing position control only; for them too, microsecond-tuned velocity and trajectory control algorithms were conceived by fine PWM dynamic generation. The adoption of an outer motion control loop, in cascade with the servo own controller, to overcome the performance degradation has been experimentally demonstrated. These techniques settled at the PIC software level also allow to measure average current consumption, by integrating instantaneous readings at 50 $\mathrm{Hz}$ duly synchronized with PWM pulses generation. From the above statements, it is clear that a door remains open to motor torque control, at least in static or pseudo-static approaches, but also in dynamic motion with additional inertial information from gyros and accelerometers.

Although being the first prototype still requiring some tuning and rectifications to reach a mature platform for research and development, interesting results on local control and pure force based balance of one leg have been already achieved. The system architecture allows for several paradigms of control, ranging from the fully centralized (inadequate from our point of view) to exclusively local to each robot joint (too complex and possibly unrealizable from our point of view) and passing by the local control capabilities modulated by global directives from the main control unit, which is the envisaged approach. Local control techniques for force, torque, position or velocity control may be based on simple traditional controllers (P, PI, PID) or fractional order controllers (Silva \& Santos, 2005), fuzzy or neural nets.

From the control point of view, the difficulty of bipedal locomotion lies in the uncertainty of the environment and the limitations of the contact between the robot and the environment (Popovic et al., 2005). Despite recent advances, accepted definitions of stability with application to the gait and posture of biped robots remain an unsolved problem and the subject of much work in robotics. The expertise of today, however, agrees on the importance of the ability to adapt efficiently to the environment as the key to enhance stability and reliability of humanoid walking. Although many papers have been published on the reflexive control in robotics, the issue of enhancing biped locomotion stability and robustness by exploiting reflexive actions has rarely been studied in the literature (Huang et al., 2005). This work explores designing and implementing fundamental local actions to deal with such events as unexpected disturbances or unknown ground irregularities. More concretely, an approach referred as adaptive leg balancing is proposed to stabilise the motion of a single leg that stands on a platform with unknown slope changes, while the control system relies on the ground reaction forces as measured by the force sensors inserted in each foot corner. The algorithm is based on the computation of the COG Jacobian transpose that provides a computationally efficient posture control algorithm less sensitive to parameter uncertainties. In order to validate the theoretical findings, most of the control schemes presented throughout the chapter are tested in several numerical simulations and experiments for representative motion tasks.

\section{The platform hardware}

Before starting the actual platform construction, several issues were considered as initial paradigms to follow. These have been: low-cost off-the-shelf components for affordability 
and easier reproducibility, full autonomy to comply with some contests' rules and also to provide independence of testing environments, upgradeability to permit initial simple approaches but with time allow the re-usage of developed modules to increase complexity, versatile control possibilities to allow for conventional but also non-conventional control methods and algorithms, distributed processing for robustness to failures and to allow growing overall computational power and also, but not the least, motion flexibility for more locomotion possibilities and approaches. Naturally, efficient power usage was also a concern and adequate power autonomy had to be sought. The global expectation was that a platform for research on control and humanoid locomotion could be developed.

\subsection{The structure design}

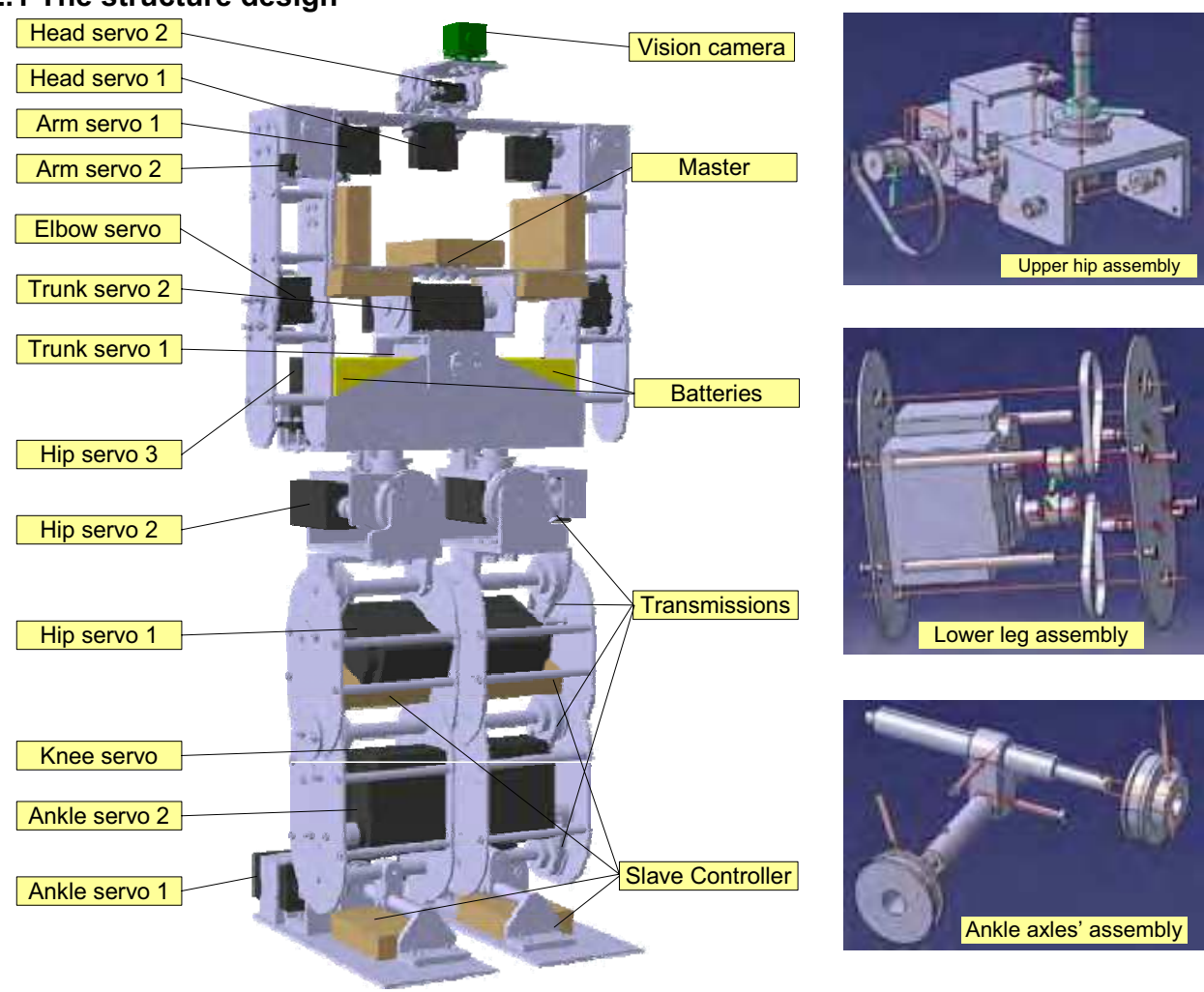

Figure 1. Three dimensional model for the humanoid structure and some assembly details

The very first concern on the platform structure was the number of degrees of freedom (DOFs). Legs were the central issue on those matters, and comparing activities and results from other research groups (mainly those present in RoboCup competitions since 2003), 6 DOFs for each leg was immediately decided. Attaching a trunk upon the hip was decided to be done with 2 DOFs. The main explanation for this decision was the expected enhanced flexibility for the moment when locomotion of the full body would arrive: having lateral and sagital compensation capabilities appeared better than only one of them. The arms were not 
given as much attention as the rest since it was expected that later on much better supported decisions could be made based on the expected know-how to come. Two degrees of freedom for the shoulder plus one for the elbow were a sufficient start. Finally, two DOFs would allow independent pan and tilt of the head, that is, of the camera expected to place on it.

The structural links to build up the platform could be done hollow, exoskeleton-like, or based on a structural internal skeleton - animal bone endoskeleton-like. Both types of solutions have been explored by other authors, but the exoskeleton approach was decided taking into account the type of actuators by-then already decided: RC servomotors; due to their volumetric geometry, they would fit better "inside" hollow links rather than "around" bone-like structures. The robot dimensions were the next concern and here the expectation was "the smallest possible" to fit all the required DOFs and remainder components. The RoboCup rules established the $60 \mathrm{~cm}$ height as the boundary between the two categories defined at the time. So it was decided: the robot had to fit the $60 \mathrm{~cm}$ height, but keeping the right proportions to be declared a compliant humanoid by RoboCup regulations. A structure was conceived from scratch and initially it was only partial. Light materials such as nylons were first tried to build the first leg but it was soon realized that aluminium would be preferable for better linkage and fixation of components along it with greater resistance to mechanical stress and strain. After some months of layout studies, and simulations, a full 3D model of the platform was reached as shown in Figure 1.

The 3D model was developed in the CATIA software and the last version accounted for circa 600 parts covering everything from bolts to belts. The model allowed testing the kinematics properties and also component clearance during locomotion. For example, one relevant issue, among several others, was the horizontal separation of the legs which imposed a trade-off between manoeuvrability when rotating upon one leg at the hip level (for which large clearance will help) and maximum torque when swapping the stance and swing legs in locomotion (for which leg horizontal proximity is an advantage). Kinematics and geometry were the first challenges to be modelled and equated. After the decision of which actuators and associated gearing would be selected, construction issues when then to the shop-floor.

\subsection{Actuators, gearing and power}

Actuation for this kind of platform is quite demanding (high torques, local feedback control needed, etc.) and off-the-shelf components do not offer much more than the popular RC servomotors. The reasons to select this type of device are commonly invoked (Ruas et al., 2006) but being sure that the servos comply with the project requisites may be difficult to predict due to their fully encapsulated behaviour and limited external controllability, since they possess a single digital control input for position setting, remaining their velocity totally beyond the control of the driving system. Hence, besides velocity control, the other concerning issue was whether their power and torque was enough to support the robot and provide locomotion. As initially only pseudo-static operation was expected, servomotors power was not a concern (velocity is small) and the attention focused on torque. Several static simulations were carried out based on the 3D model and using the real weights of motors and batteries. To compute the static torques on motors a basic formula was used (1):

$$
\tau_{k}=\left\|\sum_{i=k}^{N} m_{i} \mathbf{r}_{k i} \times \mathbf{g}\right\|
$$


where $\tau_{\mathrm{k}}$ is the torque exerted by actuator $k, m_{i}$ is the mass of link $i$ and $\mathrm{r}_{k i}$ is the vector with the relative coordinates of $i$ 's centre of mass $(\mathrm{CoM})$ relative to joint $k$ reference frame. Link numbering starts on the foot. Figure 2 illustrates the procedure with a partial example.
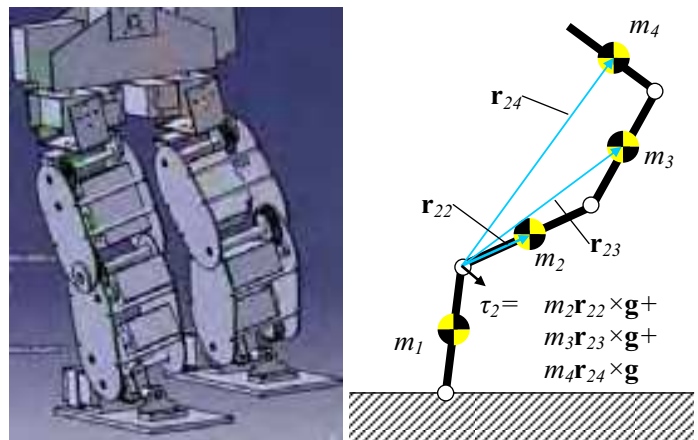

Figure 2. Calculating static torques on the Humanoid and generic example for joint 2

Table 1 shows he maximal torques predicted for each joint across the entire range of angles. The greatest torques occur on one of the foot joints and on one of the hip's joints.

\begin{tabular}{|c|c|c|c|c|}
\hline & Motor/ Joint & $\theta \min \left[{ }^{\circ}\right]$ & $\theta \max \left[{ }^{\circ}\right]$ & Tmax [N.m] \\
\hline \multirow{5}{*}{ 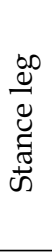 } & Foot 1 roll & -35 & +35 & 2.37 \\
\hline & Foot 1 tilt & -30 & +60 & 0.30 \\
\hline & Knee 1 & -45 & +55 & 1.17 \\
\hline & Hip 1 tilt & -60 & +60 & 0.35 \\
\hline & Hip 1 roll & -70 & +21 & 2.57 \\
\hline \multirow{5}{*}{ 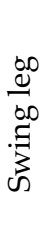 } & Foot 2 roll & -35 & +35 & 0.00 \\
\hline & Foot 2 tilt & -30 & +60 & 0.12 \\
\hline & Knee 2 & -45 & +55 & 0.30 \\
\hline & Hip 2 tilt & -60 & +60 & 0.14 \\
\hline & Hip 2 roll & -70 & +21 & 0.30 \\
\hline
\end{tabular}

Table 1. Maximal static torques on joints for the expected range of angular displacement

Off-the-shelf RC servomotors include several well-known brands, but it was found that the HITEC manufacturer implies normally lower costs. Even the strongest servomotors models from HITEC do not yield torques as large as 2.57 N.m, even when overpowered at 6.0 Volts. This implies that not all joints will be able to be direct driven by the servos. Mechanically this is a drawback because it requires gearing to increase torque (and lower the velocity). The solution adopted was to use toothed belts to connect gears with a reduction ratio of 2.5:1 (Figure 3). This increase in torque boosts the HITEC HS805BB announced 2.42 N.m to more that $5 \mathrm{~N} . \mathrm{m}$, which satisfies all static torque requirements. Moreover, it should be reminded that the torque calculations did not take advantage of trunk mobility. The trunk (and arms) was modelled as a fixed mass; with the future ability to pitch and roll the trunk, and also changing arms geometry, the CoM can be dynamically replaced and relief part of the torque applied upon some of the actuators. 


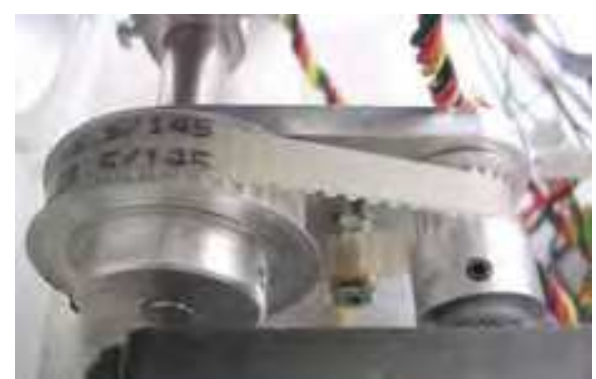

Figure 3. Transmission with a 2.5:1 gear ratio; an adjustable tensile roller holds the belt tight

As mention earlier, power to drive the motors is a most relevant issue. Servos absorb high currents, especially at start-up. Two ion-lithium batteries were installed and the system counts with a $7.2 \mathrm{~V} / 9600 \mathrm{mAh}$ pack, with maximal sustained current specified by the vendor at more than 19A. Each one of the two batteries weights circa $176 \mathrm{~g}$. Proper fusing and charge monitoring were also implemented. Figure 4 shows the selected batteries and their location on the robot.

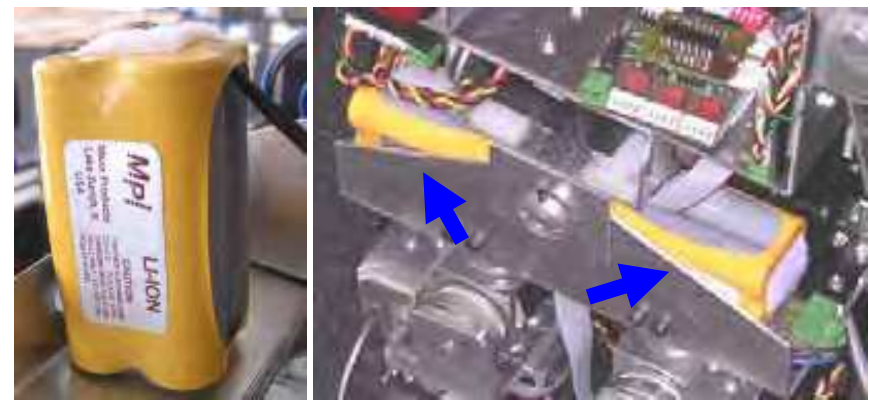

Figure 4. Batteries and their location on the robot

\subsection{Sensors and perception}

Autonomy demands perception in large extent, both of proprioceptive and exteroceptive types. For self-control and responsiveness to external perturbations due to contact, actuators and links must be monitored continuously; for awareness of the surroundings, intended for action planning or deliberate action upon the environment, some kind of remote or proximity detection is required.

The first category includes the static and dynamic status of joints which cover for angular position, but also velocity and, hopefully, torque. Moreover, measuring body interaction (force) against environment contacts can give relevant information to modify or adjust joint action to minimize some cost function during locomotion. A most meaningful example is the reaction of the floor on the feet. Indeed, if the appropriate monitoring of reaction forces on the foot sole is done, then balancing control against irregular floor may be an easier task. For this purpose, 4 force sensors were custom designed and installed on each foot. After calibration, the unbalanced force distribution among the 4 sensors on each foot may be used as input to joint actuation and permit some local control laws to rule robot behaviour, as described further. Another important issue would be to monitor the actuator torque; since that is not directly measurable (no appropriate sensors) a last attempt is to measure servos' 
current consumption; on servo motors that is not a simple task and further on some insight is given about the process.

Still on the proprioceptive sensors, inertial perception is believed to be an advantage on yetto-come developments such as dynamic locomotion patterns. Integrated Micro-ElectroMechanical (MEMS) accelerometers (ADXL202E, Analog Device) and a gyroscope (ENJ03JA, Murata) were selected and even installed, although they are not yet used to influence the system control. Finally, for the extraceptive perception, a vision system using a camera is to be mounted, although it is not yet used for decisions.

\subsubsection{Measuring joint position}

Servos have internal position feedback which is physically accessible by reading the internal potentiometer used by the device controller. However a connection must be wired to the output of the encapsulating box. Besides the tree default wires for power and input PWM pulse, a fourth wire is added to provide a voltage level between the power ground which is related to the potentiometer current position. The procedure, applicable in both the Futaba or HITEC brands (and possibly others), is sketched in Figure 5.

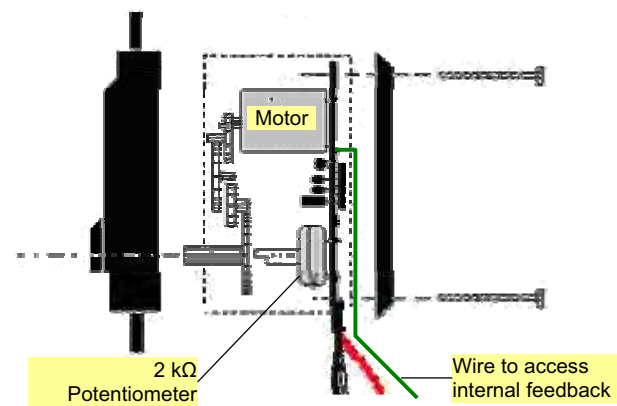

Figure 5. Wiring the servo to fetch the internal position feedback

Conditions seem now to exist to obtain joint angular position, except for the fact that voltage at the potentiometer output is not stable! Indeed, it depends on which part of the response to the PWM pulse the servo is at each moment. It was verified that the potentiometer output is only reliable during the duration of the pulse itself; once the pulse finishes, the servo internal controller enters the period of power application to the motor windings and interference will occurs as illustrated in Figure 6. A fine tuned software program had to be developed to perform potentiometer reading duly synchronized with PWM generation to ensure correct angular position evaluation.

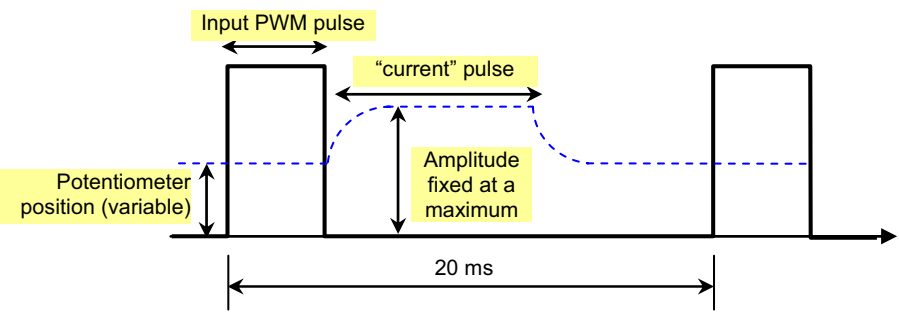

Figure 6. Value of the servomotor potentiometer along the PWM pulse sequence 


\subsubsection{Measuring current}

The procedure described in the preceding section yielded another interesting outcome which was the possibility to assess the current consumption by the servo. The "current pulse" depicted in Figure 6 appears as a "pulse" occurring immediately after PWM falling edge and its width has been related to the power applied to the motor windings; the larger the pulse the more power is applied, or, as applied voltage is constant, the more instantaneous current is being absorbed by the motor, or finally, more torque is yielded by the motor. Measuring the width of the "current pulse" was done also in synchronization with PWM pulse generation by a sampling process at a much higher frequency than the PWM pulse itself which is $50 \mathrm{~Hz}$.

\subsubsection{Measuring foot reaction forces}

To pursue a versatile platform that is expected to interact with the environment and be reactive to it, it is a must to measure contact reaction forces with the floor. This is needed to comply with floor irregularities and sloped paths, but ultimately it will provide direct feedback for balance, also in standard floor conditions. The idea is then to include the reaction forces in the control loop. Since miniature good quality load cells present prohibitive costs (hundreds of dollars), the team decided to develop low-cost strain gauge-based force sensors (Figure 7).

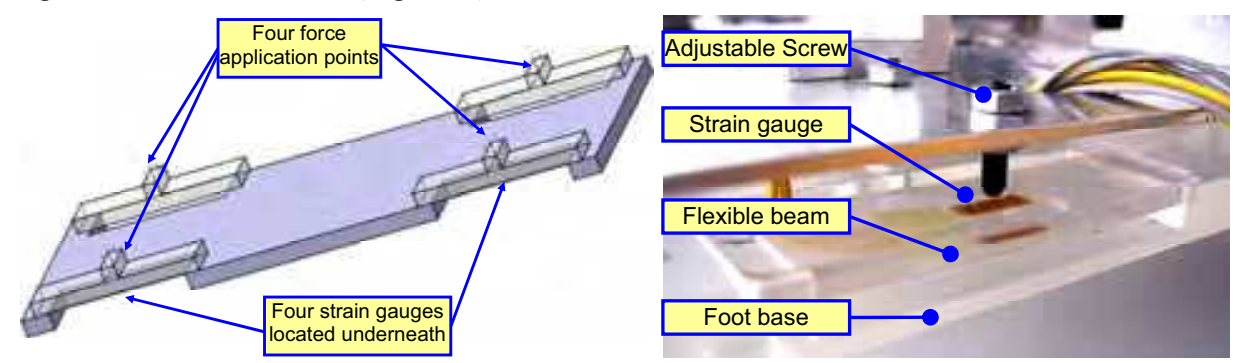

Figure 7. Model of sensitive foot and detail view of a force sensor on the foot

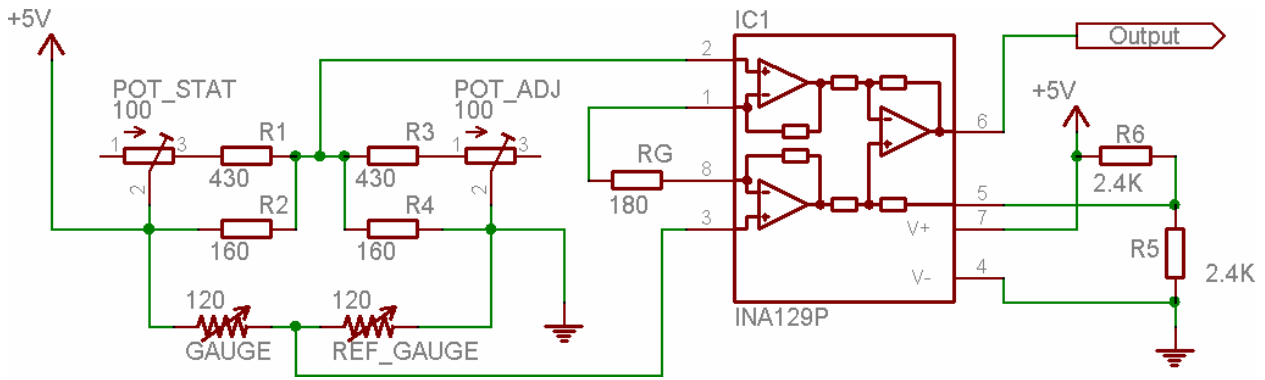

Figure 8. Electrical conditioning and amplification for the force sensor

Each foot possesses four of these sensors which allow for locomotion manoeuvres and control, either to keep the platform "upright" or even to perform dynamic balance when moving. The supporting material was made entirely of Plexiglas for greater flexibility and easier manufacture. A flexible beam of thinner Plexiglas holds the gauge which is connected to a Wheatstone bridge and an instrumentation amplifier with electrical conditioning and 
fine tuning components as shown in Figure 8. To compensate for asymmetric variations (temperature, shot noise, etc.) measuring bridge presents several points of symmetry, including a static strain gauge just for electric balancing purposes. Higher resistances than shown can be later used to low power consumption by the bridge.

\section{Distributed control architecture}

As mentioned before, the intended platform should have distributed computational capabilities either for modular and updatable software or for decentralized control capabilities in order to be more robust. Distributed architectures are not new but some systems (namely some smaller robots from those appearing in Robocup competitions) still attach to one central and global controller. The proposed approach wants to be scalable, and for so many degrees of freedom, one central controller simply is not practical.

The solution has then been to conceive a three level hierarchy of controllers: the lowest level, named Slave Units (SU), is responsible for actuator direct control and sensor reading and immediate processing; SUs may be in number of several. The second level comprises the socalled Master Unit (MU) whose role is to gather and maintain the status of the system as well as establish the communication protocols between levels. Finally, the Main Control Computer (MCC) will communicate with the MU and external interfaces, and will be responsible for the high level directives to be issued to the platform as a whole.

The Main Control, implemented as an embedded PC, will have computational power enough to acquire images and process vision. It will run a high level operating system and interfaces the MU by RS232 serial line. It issues orders of any level but does not ensure any type of real time control loop. Orders are dispatched to the Master Unit as well as querying status and other system variables, however not for real time control since there isn't even enough channel bandwidth for it. A general lay-out of the architecture appears in Figure 9. The Slave Units are indeed in charge of system motion or static activity. Each slave unit is capable of controlling three servomotors as well as acquiring sensorial data form up to 16 sensors. All slave units are connected by a CAN bus which also includes the MU.
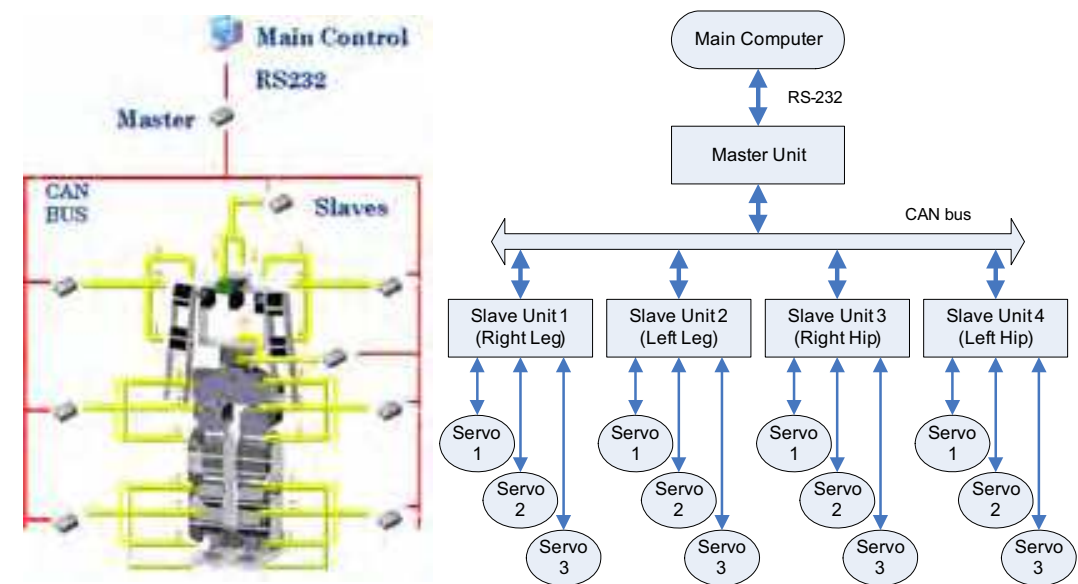

Figure 9. Concept of the distributed control architecture and one partial lay-out 
The Slave Units only send data to the bus when asked to. Their main task is to maintain some local control law for each of the 3 servos, and possibly with variable feedback depending on local sensors and/or other directives that might have reached the unit. A PIC microcontroller is the centre of the processing unit and its main components are depicted in Figure 10. All SU have conceptually the same program with variations that can dynamically be imposed depending on the SU address which is hard-coded by on-board dip-switches.

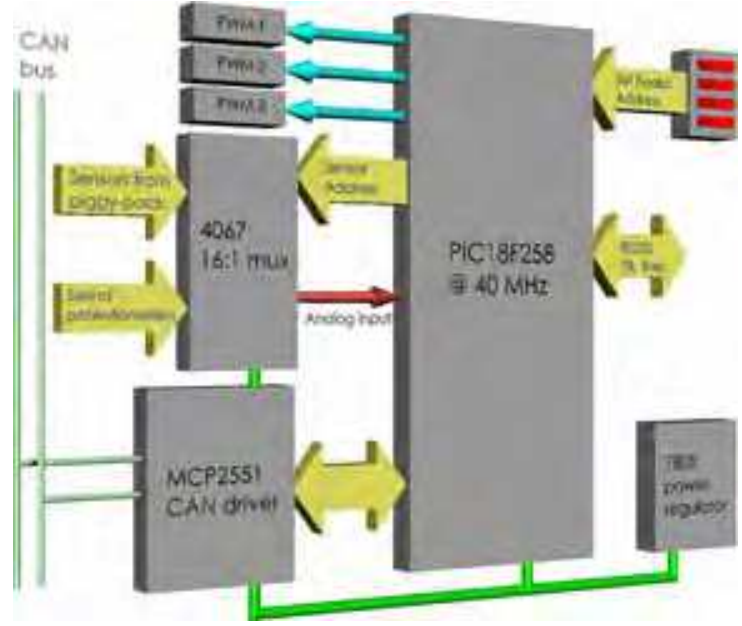

Figure 10. Functional layout of a slave controller

The Master Unit has a similar layout as slave units, but it holds a completely different program. The MU has normally no need to read any kind of sensors but it can do it if necessary. From the point of view of the hardware implementation, the basic board is similar, but a piggy-back board may be added where special sensors or other functions may be attached to a particular board (Figure 11).
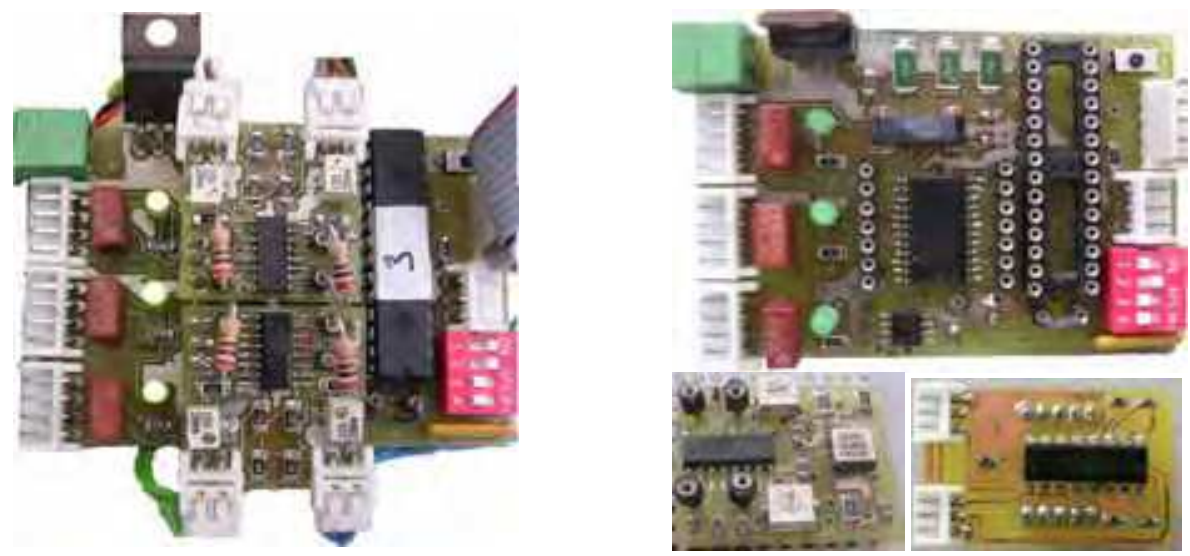

Figure 11. Complete Slave Unit (left); base board and two cases of piggy-back boards (right) 
The current stage of development of the humanoid robot designed and built in the scope of this project is shown in Figure 12. All the proposed ideas and particular control algorithms have been tested and verified on this real robot to form a critical hypothesis-and-test loop.

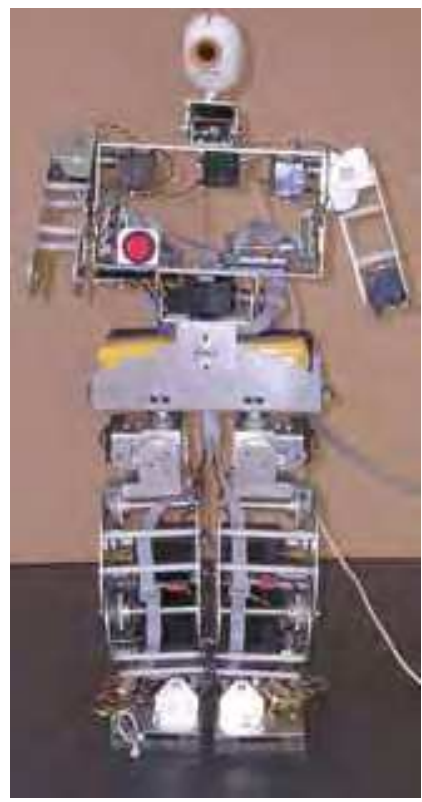

Figure 12. Biped humanoid robot with 22 DOFs

\section{Low level joint control}

Besides the computational resources, a major concern in building low-cost humanoid platforms is the implementation of the low level controllers, together with the constraints on the actuator systems. The success relies on the development of joint control algorithms and sensing devices to achieve proper performance when tracking a commanded trajectory. In this section, we will concentrate on the design and implementation of the local joint controllers. First, we review the limitations of RC servomotors with pulse-width control and how this affects our decisions. Then, we describe the implementation of an external position control loop closed around each slave unit. Adopting an outer loop, we establish a new control structure that introduces suitable compensation actions, significantly improving the system's performance and responsiveness.

\subsection{Advantages and limitations of RC servomotors}

The selected servomotors have themselves a built-in motor, gearbox, position feedback and controlling electronics, making them practical and robust devices. The control input is based on a digital signal whose pulse width indicates the desired position to be reached by the motor shaft. The internal position controller decodes this input pulse and tries to drive the motor up to the reference target based on the actual position determined by the potentiometer attached to each motor. However, the controller is not aware of the motor 
load and its velocity may vary rapidly and substantially. By design, servos drive to their commanded position fairly rapidly depending on the load, usually faster (slower) if the difference in position is larger (smaller). As the control task becomes more demanding, involving time-varying desired position (i.e., tracking control), the performance of the internal controller begins to deteriorate.

In order to validate the practical findings and gain insight into these problems, an entire system was set up intended to evaluate the actuator's performance. The experimental arrangement comprises several calibrated loads that will be applied to the servo shaft through a linkage $10 \mathrm{~cm}$ long (Figure 13). The servo is fixed in a mechanical lathe such that its zero position corresponds to the perpendicular between the link and the gravity vector.

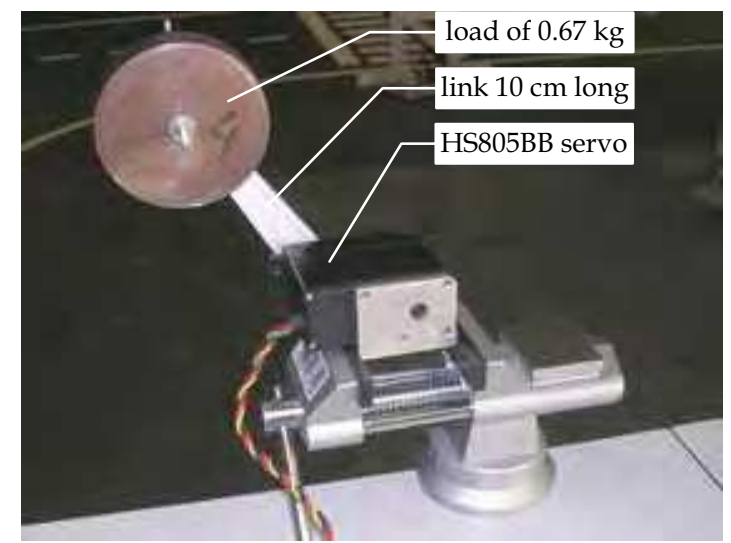

Figure 13. Experimental setup to assess servomotor response to variable loads

The setup used for experimental testing includes a master and a slave unit controlling a servomotor properly fixed and loaded. On the one side, the master unit is connected to a computer through a RS-232 link, using MatLab software as the user's interface. On the other side, the slave unit is connected to the servo mechanism in two ways: (i) by sending the desired servo position command as a pulse train with a given width; and (ii) by reading the potentiometer feedback signal (the only feedback available). In the experiments conducted below, the servo's internal controller is the only responsible for the resulting performance. In the following, results of two experiments are described: the first experiment is performed with "large" steps (equivalent to $90^{\circ}$ ) for several loads and, then, a second experiment is carried out with smaller steps (few degrees each) in order to simulate some kind of ramp input and launching the basis for velocity control.

The results of applying a step input from $-45^{\circ}$ to $+45^{\circ}$ are presented in Figure 14 in terms of the desired and the actual response for two loads (258g and $1129 \mathrm{~g})$. The first notorious observation is the unstable dynamic behaviour on position reading, which shows at the beginning a sudden jump to a position below $-45^{\circ}$ and some oscillations during the path up to the final set point. Instead, the motor shaft presented a continuous and fast motion to the final position without speed inversions or any kind of oscillations. This seems to indicate that this process also requires care since the internal controller may interfere with the voltage drop on the potentiometer that can affect external readings of the shaft position. Another problem arising from the servo response, which may be critical as the load 
increases, is the considerable steady-state errors. Notice the presence of an appreciable value of steady-sate error for the larger load (about $8^{\circ}$ error remains after the transient phase).

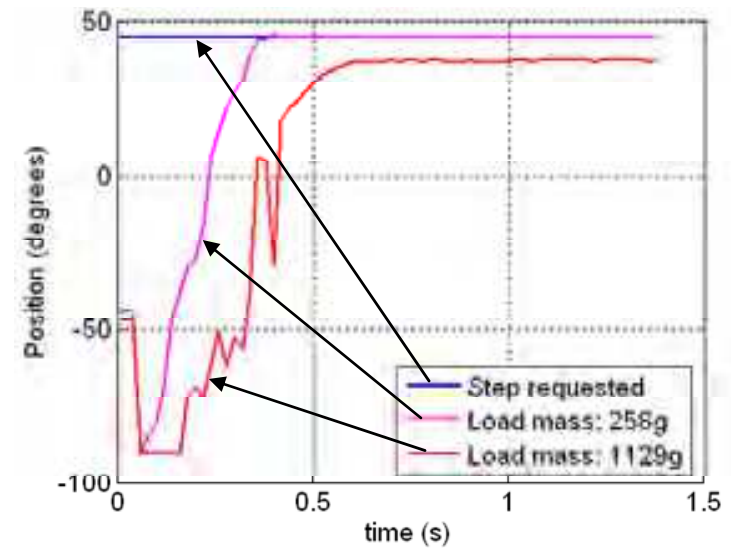

Figure 14. Response to a step input $\left(-45^{\circ}\right.$ to $\left.+45^{\circ}\right)$ in the reference

In order to carry out a fair comparison with the previous case the joint has been placed in the same initial position $\left(-45^{\circ}\right)$ and should move to the same final position $\left(+45^{\circ}\right)$. However, to implement some sort of velocity control, the experiment was carried out in a manner that small position steps are successively requested to the servo controller. Their magnitude and rate will dictate some sort of desired "average velocity". This approach will generate an approximately linear increase for the position, which is to say, some constant velocity.

The results are presented in Figure 15 in terms of the desired and the actual response to a slope input. As above, and although the transient response has a very improved behaviour, the steady state error still exists. An experiment was carried out to stress this effect: the servo is requested to successively move a given load to some positions; for each position, after motion completion, the potentiometer is sampled to obtain the real position that the servo achieved. Relating the positional error with the static torque exerted in the joint, a direct conclusion can be drawn: the higher the torque, the higher is the steady state error.

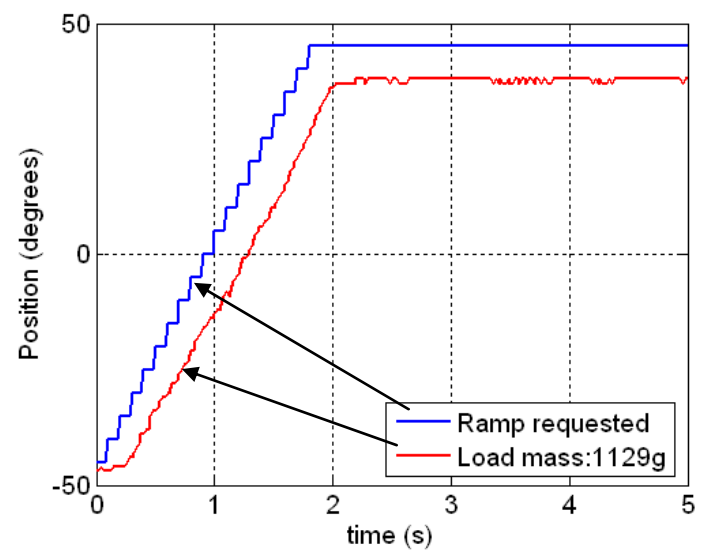

Figure 15. Response to a slope input in the reference 
In conclusion, dynamic effects and improper servo's control turns the device into a highly non-linear actuator with limited performance, which restricts the scope of their application. Two common approaches can be devised to achieve higher performance: hardware modification or software compensation. The price to pay following the first direction is, often, the replacement of the electronics unit of the motor package by dedicated control boards. On the other hand, it is expected that enhanced performance can also be achieved by software compensation, provided that position and/or torque measurements are available.

\subsection{Outer feedback control loop}

The servo circuit has a narrow input control range and it is difficult to control accurately, though it has adequate speed and torque characteristics. In most practical situations, an effective strategy to improve the servo's operation is using an external controller where an outer position control loop is closed around the inner loop available in the servomotor. Figure 16 illustrates the block diagram of the servo controller proposed to achieve enhanced performance in terms of steady-state behaviour and trajectory tracking capabilities. The algorithm is based on dynamic PWM tracking using the servo own potentiometer for position feedback. For that purpose, the slave units have to track the motor positions (up to 3 motors) with time and adjust the PWM in order to accelerate or decelerate the joint motions. Practical issues like computation time or lack of speed measurements are challenged by devising the distributed architecture approach.

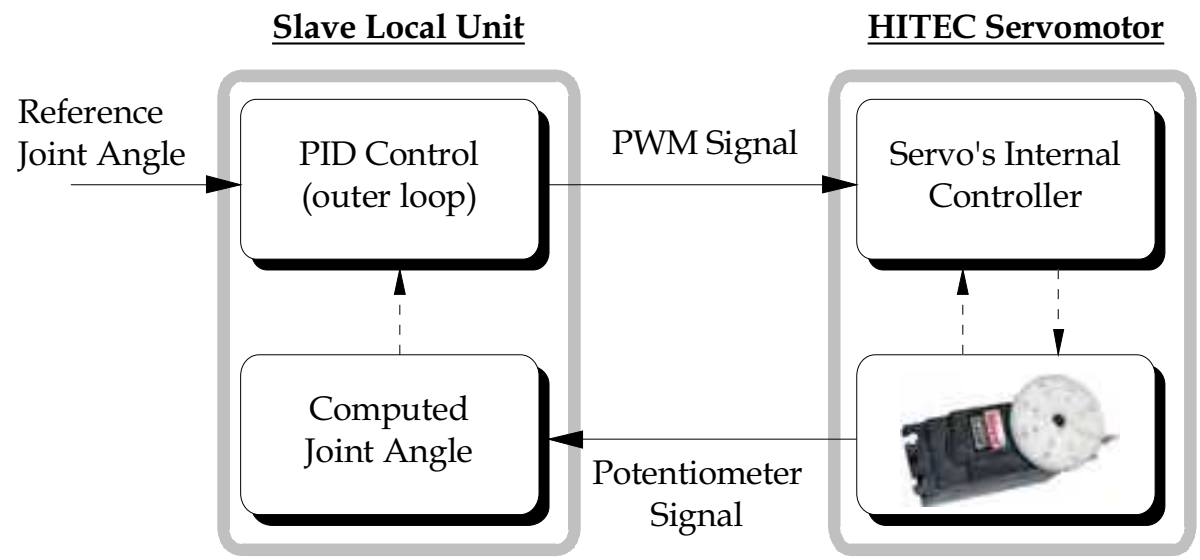

Figure 16. Block diagram of the joint control: the inner loop consists of the servo's own controller; the outer control loop generates a dynamic PWM using feedback from the servo's potentiometer

The potential offered by the external control strategy to ensure an improved behaviour is now investigated experimentally. For that purpose, several control schemes could be implemented in the PIC microcontroller. The focus of the present study is on digital PIDcontroller or any of its particular cases. The proposed control schemes are implemented in discrete time at $20 \mathrm{~ms}$ sampling interval and, then, tested in a number of experiments using the same setup as described before.

Two main considerations were made to guide the selection of the control structure. First, the system to control is formed by a single joint axis driven by an actuator with pulse-width 
control. Second, it is worth noting that an effective rejection of the steady-state errors is ensured by the presence of an integral action so as to cancel the effect of the gravitational component on the output. These facts suggest that the control problem can be solved by an incremental algorithm in which the output of the controller represents the increments of the control signal. In this line of thought, it is irrelevant the main drawback with this algorithm that cannot be used directly for a controller without integral action ( $\mathrm{P}$ or $\mathrm{PD}$ ). One advantage with the incremental algorithm is that most of the computation is done using increments only and short word-length calculations can often be used.

The first experiment is aimed at verify the effectiveness of the integral plus proportional actions. In this case, it is chosen a demanding specification for the desired slope: each new step position is update at the maximum rate of $50 \mathrm{~Hz}$ (corresponds to the PWM period) with amplitude of 5 degrees. Let the desired initial and final angular positions of the joint to be -90 and 50 degrees, respectively, with time duration of 1.12 seconds. The results are presented in Figure 17 in terms of the time history of the desired and actual angular positions, together with the trajectory errors for the full motion. It demonstrates the effect of increasing $K_{\mathrm{I}}$ for a fixed proportional term $\left(K_{\mathrm{P}}=0.04\right)$ : it reduces the lag time improving tracking accuracy, but at the expense of overshoot. Changing $K_{P}$ to a higher value $\left(K_{P}=0.30\right)$ minimises the overshoot, maintaining the lag time as for $K_{\mathrm{I}}=0.10$. From these observations, the role of each component can be deduced: (i) integral action reduces time lag at the expense of an increased overshoot; and (ii) proportional action reduces overshoot, deteriorating the establishment time for very high gains.
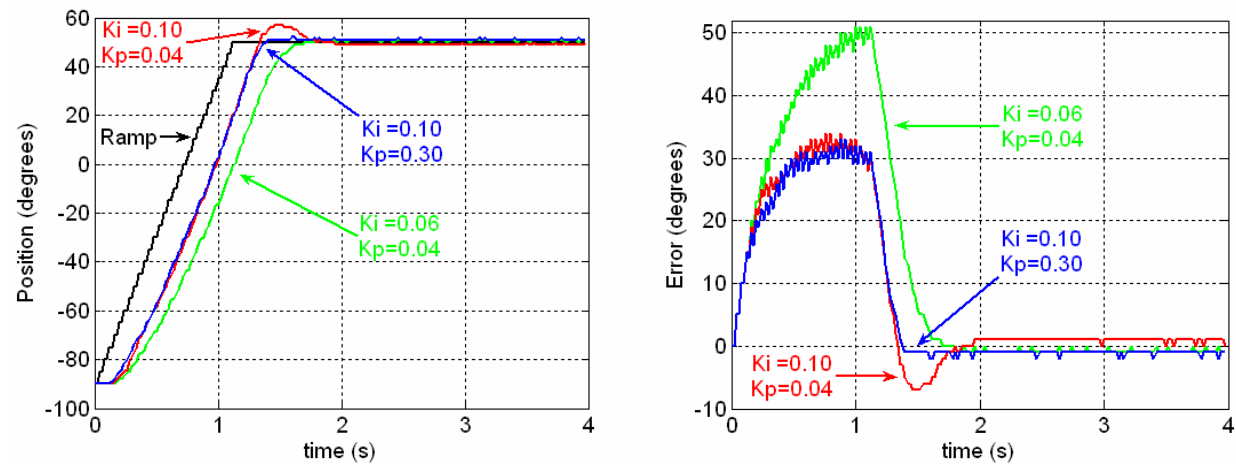

Figure 17. Behaviour of closed loop system with PI controller: the left graph shows the response to a slope input in the reference with different values of the control parameters; the right graph shows the trajectory errors for the full motion

Improvement of the position tracking accuracy might be achieved by increasing the position gain constant $K_{\mathrm{I}}$, while controlling the overshoot effects by adjusting $K_{\mathrm{P}}$. However, for high demands in terms of lag time, compensation tuning becomes very hard due to the presence of unstable oscillations during transient response. A solution to this drawback can be devised by rewrite the control algorithm aimed to include the proportional, integral and derivative terms. At the same time, the second experiment includes a planning algorithm used to generate smooth trajectories that not violate the saturation limits and do not excite resonant modes of the system. In general, it is required that the time sequence of joint variables satisfy some constraints, such as continuity of joint positions and velocities. A 
common method is to generate a time sequence of values attained by a polynomial function interpolating the desired trajectory. A third-order polynomial function in joint space was used to generate the reference trajectories. As result, the velocity has a parabolic profile and the acceleration has a linear profile with initial and final discontinuities. Figure 18 illustrates the time evolution obtained with the following initial and final conditions: $q_{\mathrm{i}}=-45^{\circ}, q_{\mathrm{f}}=45^{\circ}$, $t_{\mathrm{f}}=1.12 \mathrm{~s}$. The gains of the various control actions have been optimized by trial and error in such a way to limit tracking errors. As observed, significant improvements are achieved in the servo's response: zero steady-state error with no overshoot and limited tracking errors.

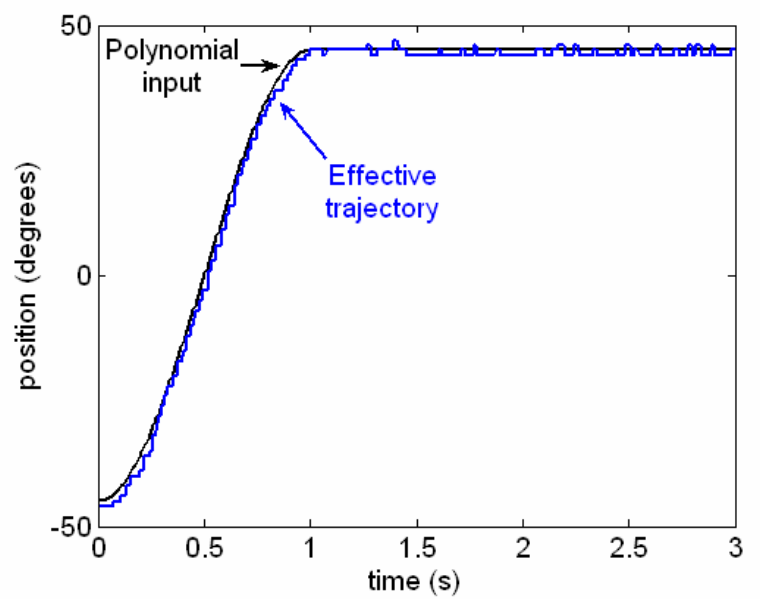

Figure 18. Behaviour of closed loop system with PID controller: the graph shows the response to a third-order polynomial joint trajectory in the reference

\subsection{Dual leg behaviour}

In this subsection, the previous control approach applied to the single-axis system is extended for the other robot's joints. Although this development phase may be facilitated by the reduction of performance demands and smaller joint excursions, the interpretation of the last results deserves attention given the influence of the driving system. The humanoid system is actuated by servomotors with reduction gears of low ratios for typically reduced joint velocities. The price to pay is the occurrence of joint friction, elasticity and backlash that contribute to the divergence between the commanded and the actual joint's position. At the lower level in the control system hierarchy lay the local controllers connected by a CAN bus to a master controller. These slave control units generate PWM waves to control three motors grouped by vicinity criteria (entire foot up to knee and hip joints) and monitor the joint angular positions by reading the servo own potentiometer. There are two servo loops for each joint control: the inner loop consists of the servo's internal controller as sold by the vendor; and the outer loop which provides position error information and is updated by the microprocessor every $20 \mathrm{~ms}$.

We now compare the robotic system's behaviour when only the inner loop is present (hereinafter "open-loop control") and when the extra feedback loop is added (hereinafter "closed-loop control"). In the later case, the outer servo loop gains are constant and tuned to perform a well-damped behaviour at a predefined velocity. Once again, the joint trajectories 
along the path are generated according to a third-order interpolating polynomial with null initial and final velocities. The next trial demonstrates the behaviour of the legs in the double-support phase, while performing basic desired movements. More concretely, the desired movements to be performed consist of: (i) a vertical motion from an upright posture; and (ii) a lateral motion in which the leg leans sideways ( \pm 27 degrees). In both cases, an additional load of $2.1 \mathrm{~kg}$ is attached to the upper part of the leg to emulate the mass of other segments (Figure 19).
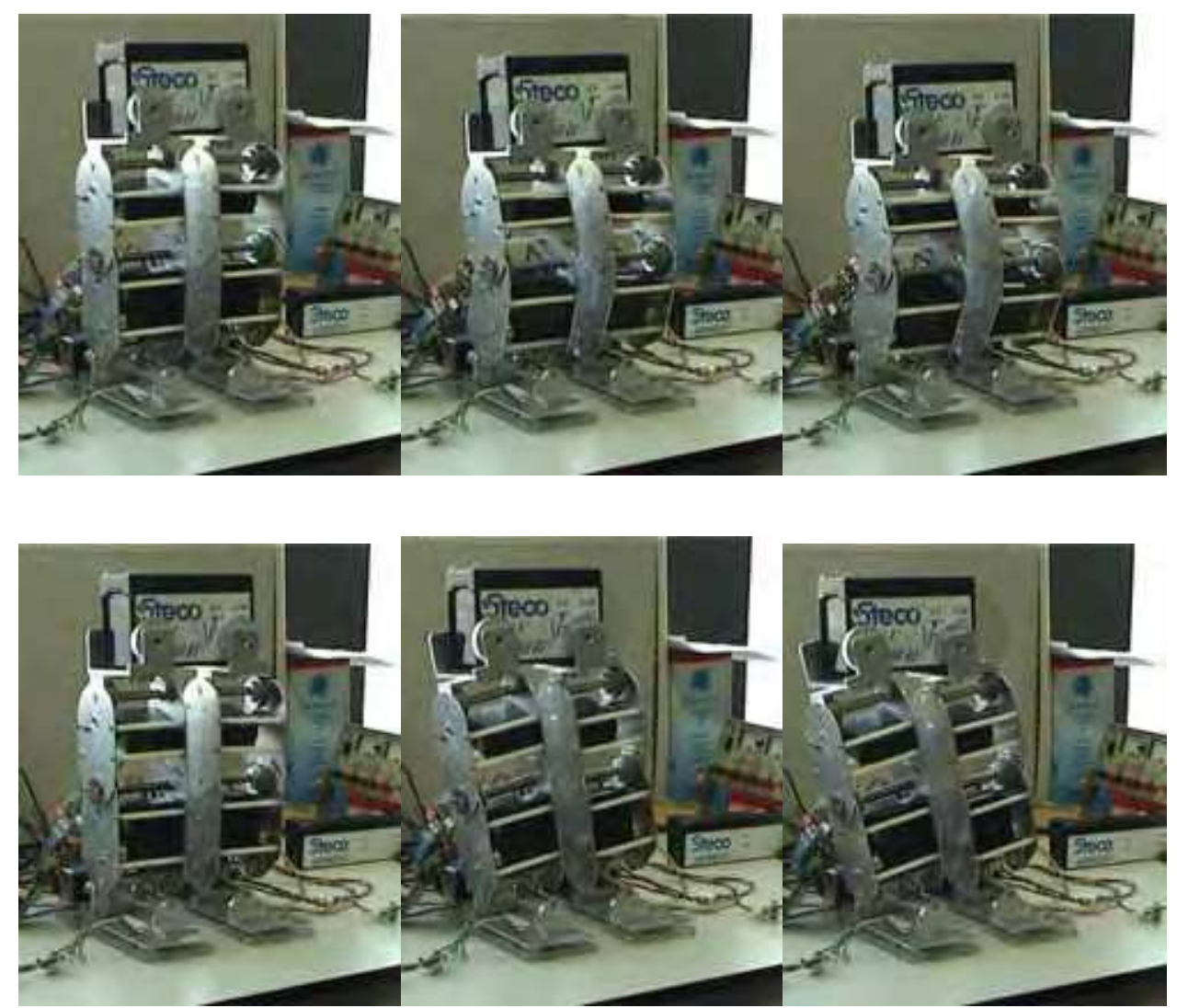

Figure 19. Snapshots of some stages in a motion sequence using two-legs and a load of 2.1 $\mathrm{kg}$ attached to the hip section: the top sequence shows the vertical motion; the bottom sequence shows the lateral motion

The experimental results in Figure 20 show the significant differences occurring in performance of the two control schemes (open-loop and the cascading close-loop controller). As expected, the open-loop control exhibits a poor performance, particularly for steady-state conditions. Due to the imposed vertical motion, the limitations of the open-loop scheme are more evident when observing the temporal evolution of the ankle (foot) joint. On the other hand, an improved performance is successfully achieved with the proposed outer control loop, both in terms of steady-state behaviour and enhanced trajectory tracking. Although 
further improvements could be possible by optimizing control gains, these results are adequate in demonstrating the efficacy of the external loop compensation approach. Finally, the performance of the servomotors is in accordance with theoretical considerations on the choice of a motor-gear combination.
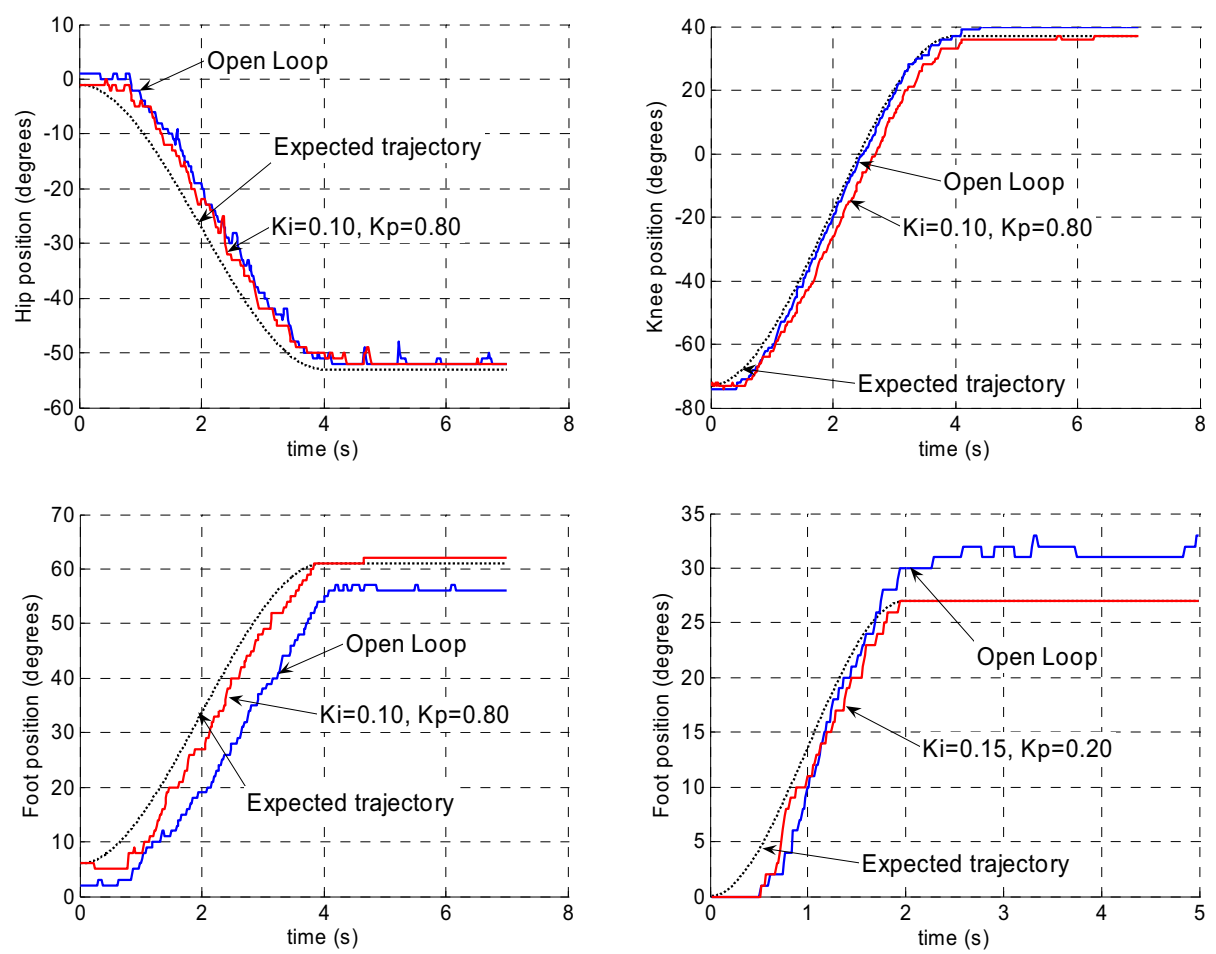

Figure 20. Comparison of performance between open and closed-loop control schemes: the top and left-bottom charts show the behaviour of the three joints during the vertical motion; the bottom-right chart shows the behaviour of the foot joint during the lateral motion

\section{Force-driven local control}

Balance maintenance is a core task for walking robots in order to engage useful tasks, ranging from standing upright posture to motion goals. The difficulty lies in the uncertainty of the environment and the limitations of the contact between the robot and the environment. Over the last years it becomes evident the dichotomy in the fundamental approaches of motion planning and control. On the one side, trajectory replaying approaches rely on accurate models of the walker being characterised by pre-planned trajectories that are played back during walking and, often, modified online through feedback (Sugihara et al., 2002; Yamasaki et al., 2002; Kajita et al., 2003). On the other side, realtime generation approaches ensure that planning and control are executed in a unified way. Gait trajectories are computed online feeding back the actual state of the system in accordance with the specified goal of the motion (Hirai et al., 1998; Denk \& Schmidt, 2001; 
Bourgeot et al., 2002). The combination of both approaches can be useful when adapting to deterministic but a priori unknown ground surfaces.

This section shows an example that is being developed to demonstrate the possibility of achieving proper humanoid leg balancing using a local control approach. To this purpose, it is considered feedback control from several sensors, including angular position in each joint and four force sensors inserted into the foot corners. The sensors provide information about the ground reaction forces and the location of the centre of pressure (COP). This opens up new avenues and possibilities for distributed architectures where centralised and local control co-exist and concur to provide robust full monitoring and efficient operation.

\subsection{Adaptive leg balancing}

The ability to balance in single support, while standing on one leg, is an important requirement for walking and other locomotion tasks. In the previous section, the approach to balance control assumed the presence of explicitly specified joint reference trajectories and calculations based on static configurations to derive the necessary PWM input signal. The goal of this section is to present the developed control algorithm that provides enhanced robustness in the control of balancing by accounting for the ground reaction forces. Thus, the system is able to stand on an uneven surface or one whose slope suddenly changes (Figure 21). In a similar way, the control system could sense that it has been pushed, using the force sensors in the soles of its foot, and acts to maintain the postural stability. The open challenge is to allow local controllers to perform control based on sensor feedback and possibly a general directive. Here, the global order is to keep balance in a desired COP location and, although all actuators can intervene, the ankle joints have the relevant role to keep an adequate force balance on each foot.

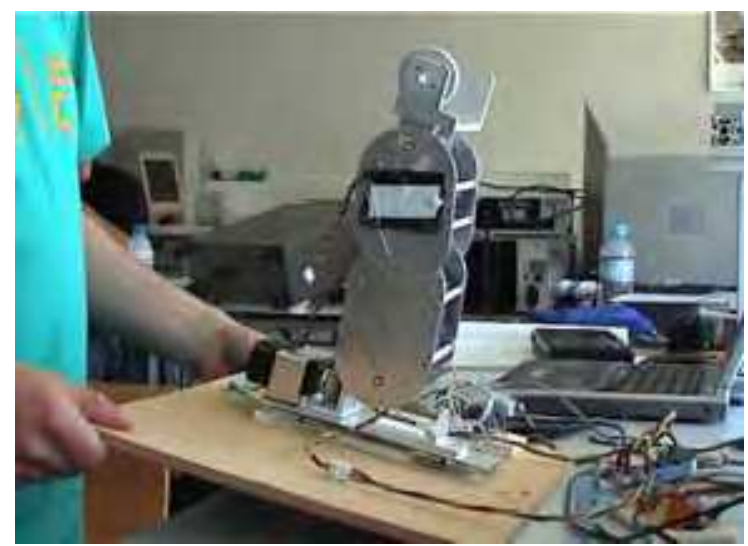

Figure 21. Single leg balancing on top of a surface with variable slope

The controller presents the following key features. First, the force sensors are used to measure the actual COP coordinates, instead of calculating other related variables, such as the centre of mass location. Second, the control system commands the joint actuators by relating the joint velocities $(\dot{q})$ to the error $(e)$ between the desired and the current position of the COP. The choice of the relationship between $\dot{q}$ and $e$ allows finding algorithms with 
different performances. The simplest method is the straightforward application of a proportional law, so that:

$$
\dot{q}=K e
$$

The controller is independent of the robot's model or any nominal joint trajectory. This approach has the main advantage of its simplicity: each component of the error vector relates directly and in an independent way to the ankle joints (pitch and roll joints), due to their orthogonal relations. Alternatively, by interpreting a small displacement in the joint vector as a torque and the error vector as a force suggests the following update law:

$$
\dot{q}=J^{T} K e
$$

Here, $\boldsymbol{J}^{T}$ is the transpose of the COG Jacobian matrix which transforms the differential variation in the joint space into the differential variation of the COG's position and $K$ is a diagonal matrix properly chosen to ensure convergence. Another requirement is now imposed in order to stabilize the hip height: the error vector accounts for the operational space error between the desired and the actual end-effector position. Then, the Jacobian translates desired Cartesian motions of selected parts of the leg into corresponding joint space motions.

\subsection{Experimental results}

The following analysis illustrates the emergence of an appropriate behaviour when the system stands on a moving platform. The desired goal is to stand in an initial posture, while the control system relies on the reaction force data to estimate slope changes in the support surface. As stated before, the emphasis in this work is on procedures that allow the robot to calibrate itself with minimal human involvement. Thus, after an initial procedure in which the humanoid leg is displaced to the desired posture, the control system generates online the necessary joint adjustments in accordance with the pre-provided goal. The joint velocity values are computed in real time to modify dynamically the corresponding PWM signal. A joint velocity saturation function is used to avoid abrupt motions, while satisfying dynamic balance constraints.

The experimental results highlight the time evolution of the COP and the resulting ankle joint angles accordingly to the control laws presented above, while the humanoid leg adapts to unpredictable slope changes. Figure 22 and Figure 23 show the achieved behaviour for a predominant leg's motion in the sagittal plane, using both the proportional and the Jacobian-based control laws. Figure 24 and Figure 25 report the leg's behaviour for a predominant motion in the lateral plane. In both cases, the use of the proposed control algorithm gives rise to a tracking error which is bounded and tends to zero at steady state. This indicates that the posture was adjusted and the differences on the ground reaction forces become small. The algorithm based on the COG Jacobian provides a computationally efficient solution for simple models. For a practical humanoid, the Jacobian may be a complex non-linear matrix requiring fast and accurate calculations using a numerical approach. Ongoing work is exploiting the case when the reference COP is a time-varying function. 

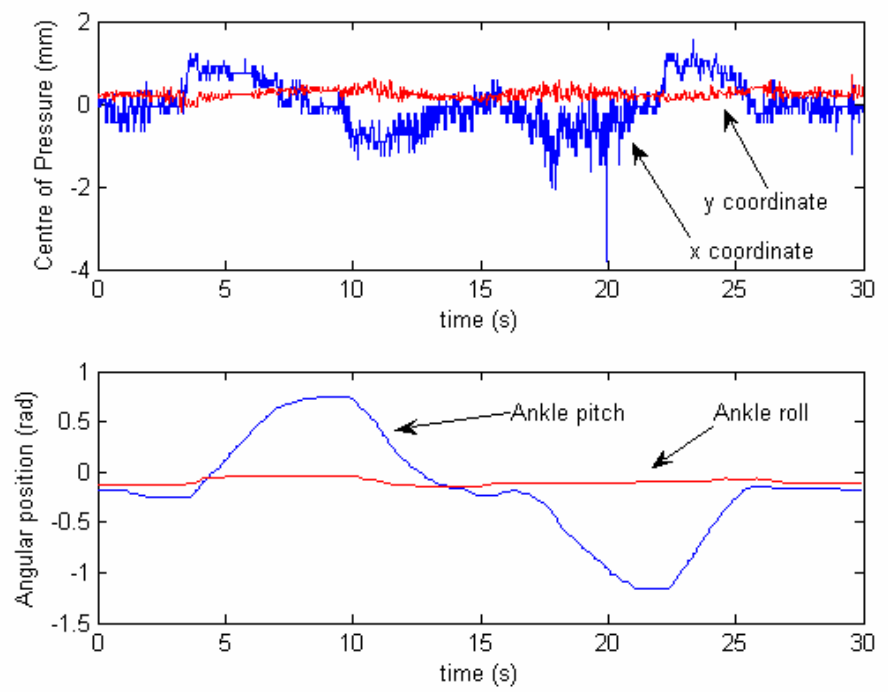

Figure 22. Leg's behaviour with predominant motion in the sagittal plane using the proportional law: temporal evolution of the centre of pressure (up) and joint angular positions (down)
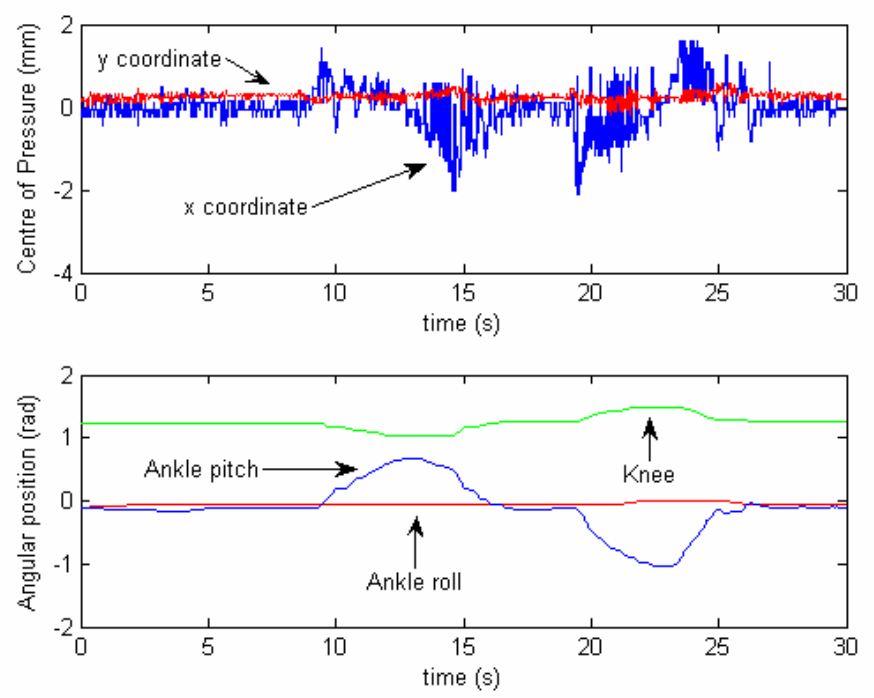

Figure 23. Leg's behaviour with predominant motion in the sagittal plane using the Jacobian-based method: temporal evolution of the centre of pressure (up) and joint angular positions (down) 

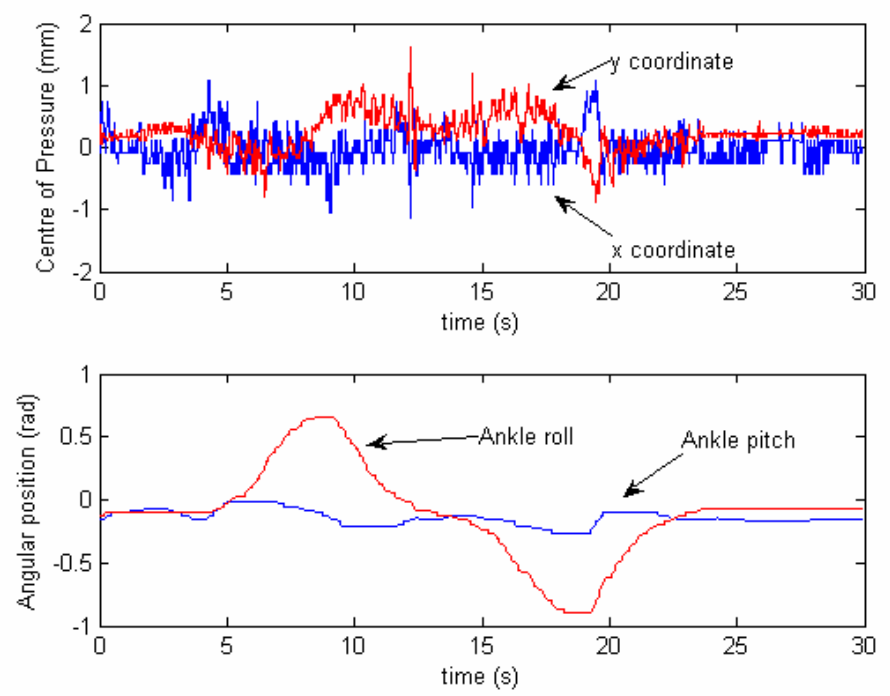

Figure 24. Leg's behaviour with predominant motion in the lateral plane using the proportional law: temporal evolution of the centre of pressure (up) and joint angular positions (down)
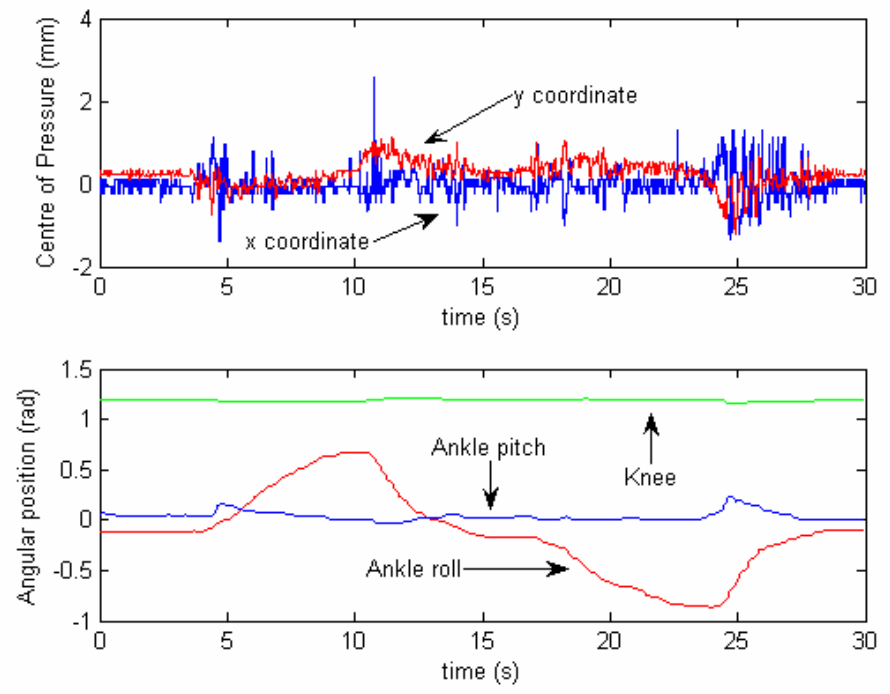

Figure 25. Leg's behaviour with predominant motion in the lateral plane using the Jacobianbased method: temporal evolution of the centre of pressure (up) and joint angular positions (down) 


\section{Conclusion}

In this chapter we have described the development and integration of hardware and software components to build a small-size humanoid robot based on off-the-shelf technologies. A modular design is conceived to ensure easy maintenance and faster reproducibility. The most relevant feature of this implementation includes the distributed architecture in which independent and self-contained control units may allow either a cooperative or a standalone operation. The integration in these simpler control units of sensing, processing and acting capabilities play a key role towards localised control based on feedback from several sensors.

The adoption of an outer motion control loop to provide accurate trajectory tracking was presented and has been experimentally demonstrated. The strength of this approach lies in its performance, generality and overall simplicity. The humanoid platform reached a point where intermediate and high level control can now flourish. An example has been given for a kind of intermediate level control implemented as a local controller. From there, a forcedriven actuation was successfully applied to demonstrate the possibility of keeping the humanoid robot in upright balance position using the ground reaction forces.

Ongoing developments on the humanoid platform cover the remainder hardware components, namely the inclusion of vision and its processing, possibly with a system based on PC104 or similar. A full autonomous humanoid robot for research is being developed that allows testing and evaluation of new ideas and concepts in both hardware and software modules. Future research, which has already started, will cover distributed control, alternative control laws and also deal with issues related to navigation of humanoids and, hopefully, cooperation. Force control techniques and more advanced algorithms such as adaptive and learning strategies will certainly be a key issue for the developments in periods to come in the near future.

\section{Acknowledgments}

The authors would like to thank the following students at the University of Aveiro for their support in the humanoid hardware and software development: David Gameiro, Filipe Carvalho, Luis Rego, Renato Barbosa, Mauro Silva, Luis Gomes, Ângelo Cardoso, Nuno Pereira and Milton Ruas.

\section{References}

Bourgeot, J.-M., Cislo, N. \& Espiau, B. (2002). Path-planning and Tracking in a 3D Complex Environment for an Anthropomorphic Biped Robot, Proceedings of the IEEE International Conference on Intelligent Robots and Systems, pp. 2509-2514, October 2002, Lausanne, Switzerland.

Denk, J. \& Schmidt, G. (2001). Synthesis of a Walking Primitive Database for a Humanoid Robot Using Optimal Control Techniques, Proceedings of the IEEE International Conference on Humanoid Robots, pp. 319-326, November, 2001, Waseda, Japan.

Furuta, T. et al. (2001). Design and Construction of a Series of Compact Humanoid Robots and Development of Biped Walk Control Strategies, Robotics and Automation Systems, Vol. 37, pp. 81-100. 
Hirai, K. et al. (1998). The Development of Honda Humanoid Robot, Proceedings of the IEEE International Conference on Robotics and Automation, pp. 1321-1326, May 1998, Leuven, Belgium.

Huang, Q., Nakamura, Y. (2005). Sensory Reflex Control for Humanoid Walking, IEEE Transactions on Robotics, Vol. 21, nº. 5, pp. 977-984.

Lohmeier, S. et al. (2004). Computer System and Control of Biped "Johnnie", Proceedings of the IEEE International Conference on Robotics and Automation, pp. 4222-4227, AprilMay 2004, New Orleans, USA.

Kajita, S. et al. (2003). Resolved Momentum Control: Humanoid Motion Planning Based on the Linear Angular Momentum, Proceedings of the IEEE International Conference on Intelligent Robots and Systems, pp. 1644-1650, October 2003, Las Vegas, USA.

Kaneko, K et al. (2004). Humanoid Robot HRP-2, Proceedings of the IEEE International Conference on Robotics and Automation, pp. 1083-1090, April-May 2004, New Orleans, USA.

Kim, J.-H. et al. (2004). Humanoid Robot HanSaRam: Recent Progress and Developments, Journal of Comp. Intelligence, Vol 8, nº. 1, pp. 45-55.

Nagasaka, K. et al. (2004). Integrated Motion Control for Walking, Jumping and Running on a Small Bipedal Entertainment Robot, Proceedings of the IEEE International Conference on Robotics and Automation, pp. 3189-3194, April-May 2004, New Orleans, USA.

Popovic, M., Goswami, A. \& Herr, H. (2005). Ground Reference Points in Legged Locomotion: Definitions, Biological Trajectories and Control Implications, The International Journal of Robotics Research, Vol. 24, n. 12, pp. 1013-1032.

Ruas, M., Silva, F. \& Santos, V. (2006). Techniques for Velocity and Torque Control of RC Servomotors for a Humanoid Robot, Proceedings of the $9^{\text {th }}$ International on Climbing and Walking Robots, pp. 636-642, September 2006, Brussels, Belgium.

Sakagami, Y. et al. (2002). The Intelligent ASIMO: System Overview and Integration, Proceedings of the IEEE International Conference on Intelligent Robots and Systems, pp. 2478-2483, October 2002, Lausanne, Switzerland.

Sugihara, T., Nakamura, Y. \& Inoue, H. (2002). Realtime Humanoid Motion Generation Through ZMP Manipulation Based on Inverted Pendulum Control, Proceedings of the IEEE International Conference on Robotics and Automation, pp. 1404-1409, May 2002, Washington, USA.

Silva, F. \& Santos, V. (2005). Towards an Autonomous Small-Size Humanoid Robot: Design Issues and Control Strategies, Proceedings of the IEEE International Symposium on Computational Intelligence in Robotics and Automation, June 2005, Espoo, Finland.

Yamasaki, F. et al. (2000). PINO the Humanoid: A Basic Architecture, Proceedings of the International Workshop on RoboCup, August-September 2000, Melbourne, Australia.

Yamasaki, F. et al. (2002). A Control Method for Humanoid Biped Walking with Limited Torque, In: RoboCup 2001, A. Birk, S. Coradeshi \& S. Tadokoro, (Ed.), pp. 60-70, Springer Verlag, Berlin Heidelberg. 


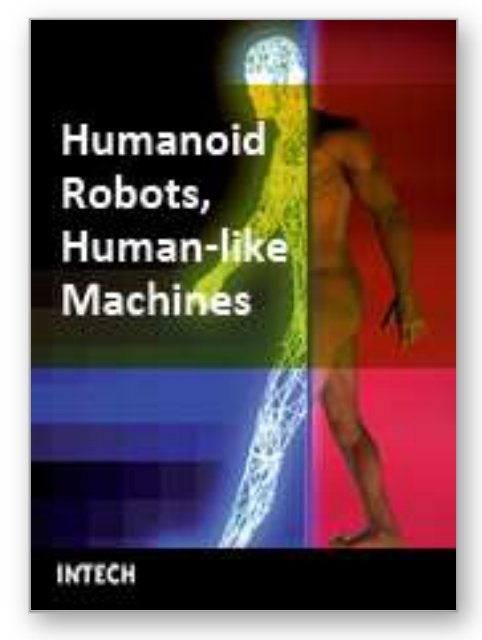

\author{
Humanoid Robots, Human-like Machines \\ Edited by Matthias Hackel
}

ISBN 978-3-902613-07-3

Hard cover, 642 pages

Publisher I-Tech Education and Publishing

Published online 01, June, 2007

Published in print edition June, 2007

In this book the variety of humanoid robotic research can be obtained. This book is divided in four parts: Hardware Development: Components and Systems, Biped Motion: Walking, Running and Self-orientation, Sensing the Environment: Acquisition, Data Processing and Control and Mind Organisation: Learning and Interaction. The first part of the book deals with remarkable hardware developments, whereby complete humanoid robotic systems are as well described as partial solutions. In the second part diverse results around the biped motion of humanoid robots are presented. The autonomous, efficient and adaptive two-legged walking is one of the main challenge in humanoid robotics. The two-legged walking will enable humanoid robots to enter our environment without rearrangement. Developments in the field of visual sensors, data acquisition, processing and control are to be observed in third part of the book. In the fourth part some "mind building" and communication technologies are presented.

\title{
How to reference
}

In order to correctly reference this scholarly work, feel free to copy and paste the following:

Filipe Silva and Vítor Santos (2007). Multipurpose Low-Cost Humanoid Platform and Modular Control Software Development, Humanoid Robots, Human-like Machines, Matthias Hackel (Ed.), ISBN: 978-3-902613-07-3, InTech, Available from:

http://www.intechopen.com/books/humanoid_robots_human_like_machines/multipurpose_lowcost_humanoid_platform_and_modular_control_software_development

\section{INTECH}

open science | open minds

\section{InTech Europe}

University Campus STeP Ri

Slavka Krautzeka 83/A

51000 Rijeka, Croatia

Phone: +385 (51) 770447

Fax: +385 (51) 686166

www.intechopen.com

\section{InTech China}

Unit 405, Office Block, Hotel Equatorial Shanghai

No.65, Yan An Road (West), Shanghai, 200040, China

中国上海市延安西路65号上海国际贵都大饭店办公楼 405 单元

Phone: +86-21-62489820

Fax: +86-21-62489821 
(C) 2007 The Author(s). Licensee IntechOpen. This chapter is distributed under the terms of the Creative Commons Attribution-NonCommercial-ShareAlike-3.0 License, which permits use, distribution and reproduction for non-commercial purposes, provided the original is properly cited and derivative works building on this content are distributed under the same license. 